\title{
Enfermedades respiratorias laborales en Chile. Ley 16.744
}

\author{
GUSTAVO CONTRERAS T.*, BERNARDITA TORREALBA J.** y MAURICIO SALINAS F.***
}

\section{Work related respiratory diseases in Chile - Law 16.744}

Since 1968 Chile has two separate systems to manage the health problems of Chileans. One is devoted to prevent and care for all kind of diseases, from gestation to death, and the other one is devoted exclusively to work-related diseases and accidents. The last one has been very successful in lowering the work related accidents below 5\%, rate that is similar to those of developed countries. The system has been replicated in other Latin-American countries. Occupational diseases, on the other hand, had been neglected by the system (although not in its design). The current article visit the most relevant aspects of the system and focus on the role of respiratory diseases specialists in the diagnosis and management of work related respiratory diseases.

Key words: Occupational diseases, lung diseases, legal aspects.

\section{Resumen}

Desde 1968 Chile cuenta con dos sistemas paralelos para manejar los problemas de salud de la población. Uno está orientado a prevenir y curar todo tipo de enfermedades, desde la gestación hasta la muerte; el otro está diseñado para preocuparse de las enfermedades y accidentes relacionados con la actividad laboral. Este último sistema ha sido muy exitoso en disminuir la accidentabilidad laboral, la que se ubica actualmente bajo el 5\%, frecuencia similar a la observada en países desarrollados. Este sistema ha sido copiado en otros países de Latinoamérica. Las enfermedades ocupacionales han sido descuidadas por el sistema, aun cuando fueron adecuadamente consideradas en el cuerpo legal. El presente artículo presenta los aspectos más relevantes de este sistema y destaca el papel de los especialistas en enfermedades respiratorias en el diagnóstico y manejo de las enfermedades respiratorias ocupacionales.

Palabras clave: Enfermedades ocupacionales, enfermedades respiratorias, aspectos legales.

Desde el $1^{\circ}$ de febrero de 1968 , Chile cuenta con dos sistemas paralelos para la atención de los problemas de salud de la población. El creado en esa fecha está específicamente orientado a prevenir y curar accidentes y enfermedades provocadas por la actividad productiva de las personas. El resto de las patologías, denominadas "comunes", están cubiertas por el Sistema Nacional de Servicios de Salud, los Institutos de Salud Previsional (Isapres) y el Fondo Nacional de Salud (Fonasa).

El sistema creado por la Ley 16.744 que "Establece Normas Sobre Accidentes del Trabajo y
Enfermedades Profesionales" ha sido tan exitoso en lograr una reducción drástica de la accidentalidad en Chile, que ha sido adoptado por países vecinos como Argentina, Colombia y Perú.

En lo medular, esta Ley crea un seguro financiado por los empleadores con un monto equivalente al $0,9 \%$ del sueldo mensual de cada trabajador contratado (Artículo 15, Ley 16.744. Tabla 1), el que debe prevenir y curar en forma oportuna y eficaz cualquier accidente o enfermedad causada por la actividad laboral.

La Ley 16.744 tiene peculiaridades que son

\footnotetext{
* Unidad de Salud Ocupacional, Clínica Río Blanco.

** Servicio de Medicina del Trabajo. Hospital del Trabajador, Asociación Chilena de Seguridad.

*** Instituto Nacional del Tórax. Facultad de Medicina Universidad de Chile.
} 
Tabla 1. Artículos seleccionados de la Ley 16.744

\section{Artículo Contenido}

Art. 5 Para los efectos de esta ley se entiende por accidente del trabajo toda lesión que una persona sufra a causa o con ocasión del trabajo, y que le produzca incapacidad o muerte. Son también accidentes del trabajo los ocurridos en el trayecto directo, de ida o regreso, entre la habitación y el lugar de trabajo. Se considerarán también accidentes del trabajo los sufridos por dirigentes de instituciones sindicales a causa o con ocasión del desempeño de sus cometidos gremiales. Exceptúanse los accidentes debidos a fuerza mayor extraña que no tenga relación alguna con el trabajo y los producidos intencionalmente por la víctima. La prueba de las excepciones corresponderá al organismo administrador

Art. 7 Es enfermedad profesional la causada de una manera directa por el ejercicio de la profesión o el trabajo que realice una persona y que le produzca incapacidad o muerte. El reglamento enumerará las enfermedades que deberán considerarse como profesionales. Esta enumeración deberá revisarse, por lo menos cada tres años. Con todo, los afiliados podrán acreditar ante el respectivo organismo administrador el carácter profesional de alguna enfermedad que no estuviere enumerada en la lista a que se refiere el inciso anterior y que hubiesen contraído como consecuencia directa de la profesión o del trabajo realizado. La resolución que al respecto dicte el organismo administrador será consultada ante la Superintendencia de Seguridad Social, la que deberá decidir dentro del plazo de tres meses con informe del Servicio Nacional de Salud

Art.15 El Seguro de Accidentes del Trabajo y Enfermedades Profesionales se financiará con los siguientes recursos:

a) Con una cotización básica general del 0,9\%, de las remuneraciones imponibles, de cargo del empleador;

b) Con una cotización adicional diferenciada en función de la actividad y riesgo de la empresa o entidad empleadora, la que será determinada por el Presidente de la República y no podrá exceder de un 3,4\% de las remuneraciones imponibles, que también será de cargo del empleador, y que se fijará sin perjuicio de lo dispuesto en el artículo 16;

c) Con el producto de las multas que cada organismo administrador aplique en conformidad a la presente ley;

d) Con las utilidades o rentas que produzca la inversión de los fondos de reserva, y

e) Con las cantidades que les corresponda por el ejercicio del derecho de repetir de acuerdo con los artículos 56 y 69

Art. 29 La víctima de un accidente del trabajo o enfermedad profesional tendrá derecho a las siguientes prestaciones, que se otorgarán gratuitamente hasta su curación completa o mientras subsistan los síntomas de las secuelas causadas por la enfermedad o accidente:

a) Atención médica, quirúrgica y dental en establecimientos externos o a domicilio;

b) Hospitalización si fuere necesario, a juicio del facultativo tratante;

c) Medicamentos y productos farmacéuticos;

d) Prótesis y aparatos ortopédicos y su reparación;

e) Rehabilitación física y reeducación profesional, y

f) Los gastos de traslado y cualquier otro que sea necesario para el otorgamiento de estas prestaciones.

También tendrán derecho a estas prestaciones médicas los asegurados que se encuentren en la situación a que se refiere el inciso final del artículo $5^{\circ}$ de la presente ley

difíciles de entender cuando ella se interpreta fuera del contexto histórico. Por ejemplo, ella separa los accidentes de las enfermedades creando un error conceptual y trastocando la importancia relativa de ambos fenómenos. Los accidentes no son más que un tipo particular de enfermedad, por lo que desde el punto de vista epidemiológico, no se justifica darles un tratamiento especial. La separación creada por la Ley determinó que el sistema se focalizara en la prevención de los accidentes y recuperación rápida del accidentado (aspecto en el que ha sido muy exitosa), pero ha relegado a un segundo plano a las enfermedades ocupacionales que afectan a muchos más trabajadores. También explica por qué este tipo de enfermedades son desconocidas para la mayor parte de la población.

La separación de los accidentes y enfermedades se debe a que en las décadas previas a la Ley, los accidentes en el trabajo afectaban a $3 \mathrm{de}$ cada 10 trabajadores, dejándolos incapacitados por largos períodos de tiempo. Ante tan alta frecuencia de eventos, las compañías de seguros de esa época cobraban tasas muy altas a los empleadores. Es así que éstos decidieron agruparse en asociaciones que comenzaron a prevenir y curar estos accidentes. Las mutualidades privadas conocidas hoy en día (Asociación Chilena de Seguridad-ACHS-, Mutual de la Cámara Chilena de la Construcción-Mutual-y el Instituto de Seguridad de Trabajo-IST), se formaron antes de que se decretara la Ley 16.744, y ella se hizo 
Art.31 El subsidio se pagará durante toda la duración del tratamiento, desde el día que ocurrió el accidente o se comprobó la enfermedad, hasta la curación del afiliado o su declaración de invalidez

La duración máxima del período del subsidio será de 52 semanas, el cual se podrá prorrogar por 52 semanas más cuando sea necesario para un mejor tratamiento de la víctima o para atender a su rehabilitación. Si al cabo de las 52 semanas o de las 104, en su caso, no se hubiere logrado la curación, y/o rehabilitación de la víctima, se presumirá que presenta un estado de invalidez

Art. 38 Si la disminución de la capacidad de ganancia es igual o superior a un $40 \%$ e inferior a un $70 \%$ el accidentado o enfermo tendrá derecho a una pensión mensual, cuyo monto será equivalente al $35 \%$ del sueldo base

Art. 39 Se considerará inválido total a quien haya sufrido una disminución de su capacidad de ganancia, presumiblemente permanente, igual o superior a un 70\%. El inválido total tendrá derecho a una pensión mensual, equivalente al $70 \%$ de su sueldo base

Art. 40 Se considerará gran inválido a quien requiere del auxilio de otras personas para realizar los actos elementales de la vida. En caso de gran invalidez la víctima tendrá derecho a un suplemento de pensión, mientras permanezca en tal estado, equivalente a un $30 \%$ de su sueldo base

Art. 58 La declaración, evaluación, reevaluación y revisión de las incapacidades permanentes serán de exclusiva competencia de los Servicios de Salud. Sin embargo, respecto de los afiliados a las Mutualidades, la declaración, evaluación, reevaluación y revisión de las incapacidades permanentes derivadas de accidentes del trabajo corresponderá a estas instituciones

Las resoluciones de las Mutualidades que se dicten sobre las materias a que se refiere este artículo se ajustarán, en lo pertinente, a las mismas normas legales y reglamentarias aplicables a los otros administradores del seguro de esta Ley

Art. 71 Los afiliados afectados de alguna enfermedad profesional deberán ser trasladados, por la empresa donde prestan servicios, a otras faenas donde no están expuestos al agente causante de la enfermedad Los trabajadores que sean citados para exámenes de control por los servicios médicos de los organismos administradores, deberán ser autorizados por su empleador para su asistencia, y el tiempo que en ello utilicen será considerado como trabajado para todos los efectos legales

Las empresas que exploten faenas en que trabajadores suyos puedan estar expuestos al riesgo de neumoconiosis, deberán realizar un control radiográfico semestral de tales trabajadores

Art. 76 La entidad empleadora deberá denunciar al organismo administrador respectivo, inmediatamente de producido, todo accidente o enfermedad que pueda ocasionar incapacidad para el trabajo o la muerte de la víctima. El accidentado o enfermo, o sus derecho-habientes, o el médico que trató o diagnosticó la lesión o enfermedad, como igualmente el Comité Paritario de Higiene y Seguridad, tendrán, también, la obligación de denunciar el hecho en dicho organismo administrador, en el caso de que la entidad empleadora no hubiere realizado la denuncia. Las denuncias mencionadas en el inciso anterior deberán contener todos los datos que hayan sido indicados por el Servicio Nacional de Salud. Los organismos administradores deberán informar al Servicio Nacional de Salud los accidentes o enfermedades que les hubieren sido denunciados y que hubieren ocasionado incapacidad para el trabajo o la muerte de la víctima, en la forma y con la periodicidad que señala el reglamento

cargo del grave problema que enfrentaban los empleadores producto de los accidentes. La tasa de accidentalidad que hoy exhibe Chile es comparable a la de los países más desarrollados del mundo ${ }^{2}$. Esta ley también dio origen a una mutual del Estado, conocida ahora como el Instituto de Seguridad Laboral.

La Ley considera accidente del trabajo aquel que se produce como consecuencia directa de la actividad productiva (lesionado por la máquina que el trabajador opera) o que ocurre durante la jornada laboral, entendida ésta desde el momento en que el trabajador abandona su casa hasta que regresa a ella (Artículo 5. Tabla 1). Si el accidente ocurre mientras se traslada entre su domicilio y el trabajo -lo que debe ser corroborado por Cara- bineros-, se denomina "accidente de trayecto"; si ocurre mientras está dentro de la empresa, aunque no esté en su puesto de trabajo, se le denomina "accidente con ocasión del trabajo". Con las enfermedades, la Ley es bastante más restrictiva, así, cataloga como "enfermedad profesional" sólo aquella generada directamente por la ocupación del afectado (Artículo 7, Ley 16.744. Tabla 1). Por lo tanto, si el panificador desarrolla asma por harina de trigo, se la cataloga como enfermedad profesional (trabaja directamente con harina de trigo), pero si la persona que enferma es la que vende el pan dentro de la misma panadería (se expone indirectamente a la harina de trigo), no se la considera enfermedad profesional.

Éstas y otras particularidades de la Ley, hacen 
que no sea fácil de entender para los profesionales encargados de aplicarla y menos para los trabajadores afectados. En este artículo procuraremos acercar la Ley 16.744 a nuestra práctica como especialistas en enfermedades respiratorias.

\section{Accidentes del trabajo}

Sin duda que el sistema respiratorio puede ser víctima de un trauma de intensidad variable, el que puede ocurrir en la empresa o durante el trayecto. Los primeros seguramente serán derivados a los servicios médicos de la aseguradora correspondiente (Artículo 76, Ley 16.744. Tabla 1), pero los segundos pueden llegar a cualquier centro asistencial donde se les debe prestar la primera atención. El primer concepto importante a considerar ante un accidente de tránsito es que alguno de los afectados puede haber estado realizando el trayecto entre su casa y su trabajo, u ocurrir como parte de su trabajo (transporte de personas o productos); es por tanto, importante recopilar esta información para cada uno de los afectados por el accidente. El segundo concepto importante es determinar si el accidente causa "días perdidos" o no. La indicación médica de reposo se vuelve aquí un elemento trascendental. Si el trauma es menor y no amerita reposo, no se lo considera accidente del trabajo. Si la indicación médica contempla uno o más días de reposo, se constituye un accidente del trabajo y es obligación del médico tratante reportar el hecho a través de un documento conocido como "Declaración Individual de Accidente del Trabajo" o DIAT (Artículo 71, Decreto Supremo 101. Tabla 2). Este documento debiera estar disponible en nuestros centros de atención y en nuestras consultas.

La decisión de indicar o no reposo, está fuertemente influenciada por el tipo de actividad que desempeña el afectado. Una fractura costal pudiera no afectar la capacidad de trabajo de un oficinista, pero sin duda limitará importantemente a un operario que debe realizar trabajo físico. Así, cuando se atiende a un accidentado, no sólo debemos indagar si el accidente fue consecuencia directa de su actividad productiva, o se produjo en la empresa mientras se encontraba fuera de su puesto de trabajo o si ocurrió en el trayecto entre el domicilio y el lugar de trabajo, sino que es fundamental conocer en detalle el tipo de actividad que desempeña el afectado.

Una vez que se haya establecido que el afectado ha sido víctima de un accidente del trabajo, se le debe derivar a la mutualidad a la que está asociada la empresa empleadora. En caso de accidentes graves, donde el traslado no es aconsejable, se debe dar aviso a la mutualidad para que ésta se haga cargo de los gastos en que incurrirá el paciente.

\section{Enfermedades profesionales}

La Ley 16.744 hace mención directa sólo a una enfermedad profesional, la silicosis, indicando una frecuencia determinada de controles radiográficos (Artículo 71, Ley 16.744. Tabla 1). El hecho de que se la referenciara, indica la importancia relativa que los legisladores concedían a esta enfermedad en esos años. Hoy sabemos que no es la enfermedad profesional más importante ni en frecuencia ni en gravedad del daño provocado al trabajador.

La Ley 16.744 se acompaña de una serie de cuerpos legales que especifican algunos aspectos que ella trata en forma general. El artículo 7 señala que un "reglamento enumerará las enfermedades que deberán considerarse como profesionales". Este reglamento es el Decreto Supremo 109, del 7 de junio de $1968^{3}$, que establece cuales son las enfermedades cubiertas por la Ley. Respecto de las enfermedades respiratorias se menciona a la Silicosis, Asbestosis, Talcosis, Beriliosis, Neumoconiosis del carbón, Bisinosis, Canabiosis, Bronquitis, Neumonitis, Enfisema y Fibrosis Pulmonar de origen químico, Asma Bronquial y el Cáncer pulmonar y de las vías respiratorias. El listado no es exhaustivo y actualmente muchas otras enfermedades han sido reconocidas como causadas por el trabajo. Considerando que la industria crece a pasos agigantados y que cada día se incorporan nuevos productos al mercado, el número de agentes capaces de generar enfermedades laborales crece diariamente. Conscientes de esta realidad, los legisladores establecieron en el cuerpo principal de la Ley (Artículo 7, Ley 16.744. Tabla 1) el mecanismo que permite incorporar patologías que no están listadas en el reglamento.

La ley 16.744 genera beneficios que no otorgan otros sistemas de salud. Por una parte, reconoce la incapacidad temporal del afectado desde el primer día de reposo, paga el subsidio por el $100 \%$ del sueldo del trabajador y extiende el beneficio hasta 2 años (Artículo 31, Ley 16.744. Tabla 1). En el caso de que la enfermedad (o accidente) cause secuelas permanentes o limite la capacidad de trabajo del afectado la Ley considera el pago de medias pensiones $-35 \%$ del sueldo base-o pensiones completas $-70 \%$ y hasta el 140\% del sueldo base- (Artículos 38 a 40, Ley 
Tabla 2. Artículo 71. Decreto Supremo 101

En caso de accidentes del trabajo o de trayecto deberá aplicarse el siguiente procedimiento:

A. Los trabajadores que sufran un accidente del trabajo o de trayecto deben ser enviados, para su atención, por la entidad empleadora, inmediatamente de tomar conocimiento del siniestro, al establecimiento asistencial del organismo administrador que le corresponda

B. La entidad empleadora deberá presentar en el organismo administrador al que se encuentra adherida o afiliada, la correspondiente Denuncia Individual de Accidente del Trabajo (DIAT), debiendo mantener una copia de la misma

C. Este documento deberá presentarse con la información que indica su formato y en un plazo no superior a $24 \mathrm{~h}$ de conocido el accidente

D. En caso que la entidad empleadora no hubiere realizado la denuncia en el plazo establecido, ésta deberá ser efectuada por el trabajador, por sus derecho-habientes, por el Comité Paritario de Higiene y Seguridad de la empresa cuando corresponda o por el médico tratante. Sin perjuicio de lo señalado, cualquier persona que haya tenido conocimiento de los hechos podrá hacer la denuncia

E. En el evento que el empleador no cumpla con la obligación de enviar al trabajador accidentado al establecimiento asistencial del organismo administrador que le corresponda o que las circunstancias en que ocurrió el accidente impidan que aquél tome conocimiento del mismo, el trabajador podrá concurrir por sus propios medios, debiendo ser atendido de inmediato

F. Excepcionalmente, el accidentado puede ser trasladado en primera instancia a un centro asistencial que no sea el que le corresponde según su organismo administrador, en las siguientes situaciones: casos de urgencia o cuando la cercanía del lugar donde ocurrió el accidente y su gravedad así lo requieran. Se entenderá que hay urgencia cuando la condición de salud o cuadro clínico implique riesgo vital y/o secuela funcional grave para la persona, de no mediar atención médica inmediata. Una vez calificada la urgencia y efectuado el ingreso del accidentado, el centro asistencial deberá informar dicha situación a los organismos administradores, dejando constancia de ello

G. Para que el trabajador pueda ser trasladado a un centro asistencial de su organismo administrador o a aquél con el cual éste tenga convenio, deberá contar con la autorización por escrito del médico que actuará por encargo del organismo administrador

H. Sin perjuicio de lo dispuesto precedentemente, el respectivo organismo administrador deberá instruir a sus entidades empleadoras adheridas o afiliadas para que registren todas aquellas consultas de trabajadores con motivo de lesiones, que sean atendidos en policlínicos o centros asistenciales, ubicados en el lugar de la faena y/o pertenecientes a las entidades empleadoras o con los cuales tengan convenios de atención. El formato del registro será definido por la Superintendencia

16.744. Tabla 1) hasta que el afectado cumpla 65 años, ya que de allí en adelante queda cubierto por la pensión de vejez. Cabe hacer notar que cuando se decretó esta Ley, el sistema previsional Chileno establecía que el monto de la jubilación era equivalente al $70 \%$ del sueldo, monto muy superior a cualquiera de las pensiones establecidas para el sistema. En caso de fallecimiento se hace cargo de la esposa e hijos por tiempos determinados.

Tan importante como lo anterior, es la cobertura de salud que considera el pago al $100 \%$ de todos los gastos médicos, quirúrgicos, dentales, en forma ambulatoria $u$ hospitalizada, incluyendo medicamentos, insumos, prótesis, aparatos ortopédicos, rehabilitación y traslados (Artículo 29 Ley 16.744. Tabla 1).

Los beneficios de cobertura de salud son los que justifican que se diagnostique correcta $y$ oportunamente todas las enfermedades respiratorias causadas por el trabajo, ya que los afectados tendrán acceso a todas las terapias que sean necesarias y sin tope alguno en gastos. La Ley busca asegurar la recuperación más completa y rápida posible del afectado.

Además, algunas enfermedades crónicas tienen la posibilidad de curar si son causadas por el trabajo. Citemos por ejemplo el asma bronquial. Si la enfermedad es causada por un agente presente sólo en el ambiente laboral (harina de trigo por ejemplo) el retiro oportuno de la exposición dará al trabajador la posibilidad de curar la enfermedad. Es el único tipo de asma que puede curar, habida consideración de que el sujeto no vuelva a exponerse al agente causante. El retiro del trabajador del puesto de trabajo que le ocasionó la enfermedad, es una de las acciones preventivo/ curativas más emblemáticas de la Ley 16.744 (Artículo 71, Ley 16.744. Tabla 1).

Al igual que para el caso de los accidentes, cada vez que nos corresponda hacer el diagnóstico de una enfermedad profesional, debemos 
declararla en el formulario ad-hoc conocido como "Declaración Individual de Enfermedad Profesional" o DIEP, y derivar al afectado a la mutualidad correspondiente.

\section{Diagnóstico de las enfermedades respiratorias profesionales}

El diagnóstico de las enfermedades profesionales tiene algunas peculiaridades producto del carácter legal que se les atribuye y de los requisitos necesarios para obtener la cobertura del seguro.

En primer lugar se debe tener presente que la “declaración, evaluación, reevaluación y revisión de las incapacidades permanentes serán de exclusiva competencia de los Servicios de Salud" (Artículo 58, Ley 16.744. Tabla 1), lo que debe interpretarse como que sólo dichos organismos tienen la facultad de declarar que un trabajador padece una enfermedad profesional que le ocasiona incapacidad permanente. Así, por ejemplo, el diagnóstico de silicosis sólo puede ser efectuado por una de las Comisiones de Medicina Preventiva e Invalidez (COMPIN) distribuidas a lo largo de Chile, por la Comisión Médica de Reclamos (COMERE) o la Superintendencia de Seguridad Social (SUSESO).

En segundo lugar, los criterios diagnósticos y de evaluación del grado de incapacidad generado por una enfermedad determinada están especificados en diversos documentos. El principal es la Circular 3G40 del 14 de marzo de $1983^{4}$ la que se complementa, en lo que se refiere a las enfermedades respiratorias provocadas por el trabajo, con el "Manual de Bronconeumopatías Ocupacionales" elaborado por el Ministerio de Salud y publicado en $1981^{5}$. Por ejemplo, si bien el diagnóstico médico de silicosis puede efectuarse mediante una biopsia pulmonar o mediante una tomografía axial computada; los cuerpos legales establecen que el diagnóstico se hará cuando la enfermedad esté visible en la radiografía de tórax ${ }^{6}$ y la historia de exposición laboral a sílice sea 'suficiente' (no indica que se entiende por 'suficiente'). Surgen aquí una serie de consideraciones respecto de si la disposición legal contenida en la Circular 3G40 es correcta o no, y al debate, nuestra especialidad podría aportar la información científica que permita sostener una u otra postura.

En 2013 el Ministerio de Salud comenzó el trabajo de actualizar el "Manual de Bronconeumopatías Ocupacionales", reemplazándolo por una "Guía de Prevención, Diagnóstico y Manejo Enfermedades Respiratorias Relacionadas con el Trabajo", que pretende incorporar los conceptos actuales sobre guías de práctica clínica.

\section{Evaluación de la incapacidad producida por las enfermedades respiratorias ocupacionales}

Las disposiciones legales no se actualizan con la velocidad que lo hace el conocimiento científico, debido al proceso administrativo que hay detrás de cada una de ellas. La misma circular 3G40 que instruye cómo realizar el diagnóstico de algunas enfermedades respiratorias, especifica cómo ha de evaluarse la incapacidad producida por ellas. Volviendo al ejemplo de la silicosis, establece que la combinación de dos parámetros constituye el eje central de la valoración del grado de incapacidad producido por la enfermedad.

Uno de los índices es la radiografía de tórax, la que debe obtenerse e informarse de acuerdo a las recomendaciones de la Organización Internacional de Trabajo (OIT) ${ }^{7}$. Las interpretaciones acerca de cómo debe entenderse esta parte de la disposición, es materia de controversia a la que nuestra especialidad podría aportar información. La importancia que se le concede a la radiografía de tórax en los programas de pesquisa de trabajadores expuestos a agentes neumoconiógenos (sílice, asbesto, carbón) es indiscutible, dado que tiene una alta sensibilidad a bajo costo. Pero la que se le otorga en la evaluación de incapacidad parece sobrevalorada. En primer lugar no se toma en cuenta la gran capacidad del pulmón para resistir el daño provocado por la silicosis, lo que explica que la enfermedad progrese desde el grado leve al avanzado en la radiografía, sin que aparezcan síntomas respiratorios. Así y todo, es la progresión radiográfica la determinante principal del grado de incapacidad provocado por la enfermedad. Más aun, es posible observar trabajadores en etapa de fibrosis masiva progresiva con enormes masas que comprometen ambos pulmones, y que por ende son catalogados también con una incapacidad mayor, absolutamente asintomáticos. Por otro lado, la interpretación de las imágenes visibles en la radiografía no es unívoca, por lo que siempre debiera recurrirse a exámenes complementarios para realizar un diagnóstico diferencial adecuado.

El otro índice utilizado para evaluar el grado de incapacidad provocado por la silicosis es la espirometría basal, valorada según las ecuaciones de Kory y cols ${ }^{8}$. Documentos oficiales posteriores establecen que se debe utilizar los valores de referencia de Knudson y cols ${ }^{9}$, aunque se reconoce éstos tampoco son adecuados para los trabajadores y trabajadoras chilenos/as. En cuanto se comenzó a utilizar las ecuaciones de Knudson y cols., aparecieron publicaciones que pusieron en evidencia la inconveniencia de utilizar valores de referencia obtenidos en población anglosajona a 
personas chilenas. En 1988 Santolaya y cols ${ }^{10}$, demostraron que los estándares internacionales no se aplicaban adecuadamente a trabajadores de Chuquicamata; resultados similares se obtuvieron en el estudio de Marambio y cols, en $1992^{11}$. En 1996, en una editorial de la Revista Médica de Chile, el Dr. Manuel Oyarzún señaló que las ecuaciones de Knudson y cols, subestiman en más de un $10 \%$ los parámetros de la espirometría en chilenos ${ }^{12}$. Más tarde (2002) Contreras y cols, en un estudio realizado población adulta del Área Metropolitana, estimaron en $20 \%$ esa diferencia ${ }^{13}$. Otros estudios mostraron resultados similares, por lo que un grupo de especialistas se abocó a la tarea de unificar los distintos esfuerzos realizados en el país, para finalmente hacer una nueva propuesta de valores de referencia para espirometría en población chilena ${ }^{14}$.

El uso de valores de referencia inadecuados genera una exigencia muy alta a trabajadores cuya espirometría basal presenta valores que oscilan entre un 120 y $150 \%$ del teórico, puesto que la circular 3G40 señala que se consideran normales valores de CVF y $\mathrm{VEF}_{1}$ a aquellos superiores a $80 \%$ del teórico. De este modo, los índices espirométricos deben deteriorarse más de un $40 \%$, para recién indicar que el trabajador sufre una incapacidad leve. Si bien la circular establece la posibilidad de considerar el deterioro en el tiempo de la espirometría como un indicador de daño ("que en un período de 5 años muestran un descenso mayor de $10 \%$ no atribuible a la edad, deben considerarse como muy probablemente debidos a los efectos del agente neumoconiógeno o fibrógeno químico o biológico"), no indica como valorar el porcentaje de caída y, por ende, en la práctica no se aplica.

Tampoco se pone suficiente atención al hecho de que las neumoconiosis afectan principalmente al parénquima pulmonar y secundariamente a las vías respiratorias. Es así posible observar extenso daño pulmonar en la radiografía de tórax y disminución significativa de la capacidad funcional del afectado, sin ninguna repercusión en la espirometría; se olvida que ella no es adecuada para evaluar un aspecto funcional tan esencial en las neumoconiosis como es la hematosis.

La Circular 3G40 también menciona que pueden utilizarse otros exámenes, como la medición de volúmenes pulmonares, capacidad de difusión de monóxido de carbono y gasometría arterial en reposo y ejercicio para evaluar el grado de daño provocado por las enfermedades respiratorias ocupacionales; sin embargo, tampoco indica qué valor conceder a los resultados de estas pruebas, y por ende, no se usan.
Acercar la evaluación de incapacidad provocada por la enfermedad respiratoria contenida en los cuerpos legales nacionales al conocimiento médico más actualizado, puede ser otra contribución de nuestra especialidad al enorme y cambiante campo de las enfermedades respiratorias ocupacionales.

\section{Agradecimientos}

Los autores agradecen la revisión crítica y comentarios del Dr. Manuel Oyarzún G.

\section{Bibliografía}

1.- LEY 16.744 Establece Normas Sobre Accidentes del Trabajo y Enfermedades Profesionales. Publicada el 1 de febrero de 1968. República de Chile.

2.- MAGAZINE 4. La prevención de accidentes laborales. Agencia Europea para la Seguridad y la Salud en Trabajo, 2002. Disponible en: https://osha.europa.eu/es/ publications/magazine/4. Consultado el 1 de marzo de 2014.

3.- DECRETO SUPREMO 109. Aprueba Reglamento para la Calificación y Evaluación de los Accidentes del Trabajo y Enfermedades Profesionales, de Acuerdo con lo Dispuesto en la Ley 16.744 , de $1^{\circ}$ de febrero de 1968 , que Estableció el Seguro Social Contra los Riesgos por estos Accidentes y Enfermedades. Publicado el 7 de junio de 1968. República de Chile.

4.- CIRCULAR $N^{\circ} 3 \mathrm{G} / 40$. Instructivo para la Calificación y Evaluación de las Enfermedades Profesionales del reglamento D.S. $N^{\circ} 109 / 1968$ de la Ley 16.744. Ministerio de Salud. República de Chile. 14 de marzo de 1983.

5.- MANUAL DE BRONCONEUMOPATÍAS OCUPACIONALES. Ministerio de Salud. República de Chile. 1981.

6.- CIRCULAR B $2 \mathrm{~N}^{\circ}$ 32. Instruye sobre Diagnóstico y Evaluación Médico-Legal de silicosis. Ministerio de Salud. República de Chile. 10 de junio de 2005.

7.- ORGANIZACIÓN INTERNACIONAL DEL TRABAJO. Guía para el Uso de la Clasificación Internacional de la OIT de Radiografías de Neumoconiosis. Edición Revisada. 2000.

8.- KORY R C, CALLAHAN R, BOREN H G, SYNER M J. The Veterans Administration-Army cooperative study of pulmonary function. I. Clinical spirometry in normal men. Am J Med 1961; 30: 243-58.

9.- KNUDSON R J, LEBOWITZ M D, HOLBERG C J, BURROWS B. Changes in the normal maximal expiratory flow-volume curve with growth and aging. Am Rev Respir Dis 1983; 127: 725-34.

10.- SANTOLAYA R, ARAYA J, ALFARO R. Inaplicabilidad de los patrones internacionales de capacidad vital 
(CV) en poblaciones adultas residentes permanentes de alturas: Chuquicamata $(2.800 \mathrm{~m})$. Revista Médica del Cobre 1988; 1: 139-49.

11. MARAMBIO J A, SANDOVAL H, VALENZUELA P, MORENO R, DONOSO H, CONTRERAS G, et al. Espirometría en población general del Gran Santiago: Comparación con los valores de referencia de Knudson. Rev Chil Enf Respir 1992; 8: 241 (Resumen CL-9-3).

12.- OYARZÚN M. Valores espirométricos normales en la población chilena. (Editorial) Rev Med Chile 1996; 124: 1365-7.
13.- CONTRERAS G, ANTOINE J, CUMSILLE F, MILLER A. Valores de referencia para espirometría en población adulta del Área Metropolitana de Chile. Rev Chil Enf Respir 2002; 18: 295 (Resumen P46).

14.- GUTIÉRREZ M, VALDIVIA G, VILLARROEL L, CONTRERAS G, CARTAGENA C, LISBOA C. Proposición de nuevos valores de referencia espirométricos para evaluar función pulmonar en población chilena adulta. Sociedad Chilena de Enfermedades Respiratorias (SER) Ecuaciones de referencia espirométricas SER: (ERE-SER)". Rev Med Chile 2014; 142: 143-52. 